\title{
Wide Variability of Pediatric Knee Arthroscopy Case Volume in Orthopaedic Surgery Residency
}

\author{
Suleiman Y. Sudah, M.D., David S. Constantinescu, M.D., Matthew H. Nasra, M.B.S., \\ Christopher R. Michel, M.D., Christopher N. Dijanic, M.D., Daniel J. Kerrigan, M.D., and \\ Ryan J. Plyler, M.D.
}

\begin{abstract}
Purpose: The purpose of this study was to evaluate orthopaedic surgery resident case volume and variability for adult and pediatric knee arthroscopy from 2016 to 2020. Methods: The Accreditation Council for Graduate Medical Education surgical case log data from 2016 to 2020 for graduating United States orthopaedic surgery residents were analyzed. The average number of total (adult and pediatric), adult, and pediatric knee arthroscopy cases were compared from 2016 to 2020. The 10th and 90th percentiles of case volumes for adult and pediatric knee arthroscopy procedures were compared from 2016 to 2020 to determine caseload variability. Results: There was an $18 \%$ increase in pediatric knee arthroscopy cases between 2016 and 2020 (average: $13.9 \pm 10$ to $16.4 \pm 13 ; P<.005$ ), a $5.4 \%$ decrease in adult knee arthroscopy cases $(100 \pm 45$ to $94.6 \pm 47 ; P<.027)$, and a $2.6 \%$ decrease in total knee arthroscopy (113.9 \pm 47 to $111 \pm 51 ; P=.264)$ cases. There was an 11 -fold difference in the number of pediatric knee arthroscopy cases performed between the 10 th and 90th percentile of residents in 2020 ( 3 vs 33 cases, respectively), a 3.28-fold difference for adult knee arthroscopy (47 vs 154, respectively), and a 2.98-fold difference for total knee arthroscopy (59 vs 176, respectively). Conclusions: Pediatric knee arthroscopy comprises a small yet growing percentage of total knee arthroscopy case volume of graduating orthopaedic surgery residents. However, wide variability in resident exposure is present and likely masked by the abundance of adult cases performed each year. Clinical Relevance: The findings presented in this study may assist in optimizing arthroscopy resident education. Existing ACL reconstruction and knee arthroscopy case minimum requirements could be updated to include a set number of pediatric cases. These changes might help reduce case volume variability and discrepancies in resident education.
\end{abstract}

\section{Introduction}

$\mathbf{M}$ ajor changes in orthopaedic residency education have occurred in the last decade. In 2013, the National Accreditation Society (NAS) implemented a set of clinical competency milestones in 16 orthopaedic clinical areas. ${ }^{1}$ Semiannual assessment of a resident's medical knowledge and procedural skill within these

From the Department of Orthopedic Surgery, Monmouth Medical Center, Long Branch, New Jersey, U.S.A. (S.Y.S., C.R.M., C.N.D., D.J.K.); Department of Orthopedic Surgery, University of Miami, Miami, Florida, U.S.A. (D.S.C); Rutgers Robert Wood Johnson Medical School, New Brunswick, New Jersey, U.S.A. (M.H.N.); and Professional Orthopedic Associates, Tinton Falls, New Jersey, U.S.A. (R.J.P.).

Full ICMJE author disclosure forms are available for this article online, as supplementary material.

Received April 26, 2021; accepted August 17, 2021.

Address correspondence to Suleiman Y. Sudah, M.D., 50 Chelsea Ave., \#7421, Long Branch, NJ 07740,USA.E-mail: sudahslay@gmail.com

(C) 2021 THE AUTHORS. Published by Elsevier Inc. on behalf of the Arthroscopy Association of North America. This is an open access article under the CC BY-NC-ND license (http://creativecommons.org/licenses/by-nc-nd/4.0/). 2666-061X/21622

https://doi.org/10.1016/j.asmr.2021.08.005 categories is sent to the Accreditation Council for Graduate Medical Education (ACGME) and used to define program outcomes. ${ }^{1}$ The ACGME has implemented a number of case minimum requirements within these areas to ensure residents gain adequate exposure.

Anterior cruciate ligament (ACL) and meniscus injury are pillars of orthopaedic surgery residency education and account for 2 of the 16 clinical competency milestones. While the ACGME mandates residents to perform a minimum of 30 knee arthroscopy and $10 \mathrm{ACL}$ reconstruction procedures each year, no distinction between pediatric and adult cases is made. However, the surgical indications, techniques, instrumentation, and operative risks associated with these procedures vary considerably among these demographics. Therefore, it is important to view pediatric and adult knee arthroscopy as individual entities.

The purpose of this study was to evaluate orthopaedic surgery resident case volume and variability for adult and pediatric knee arthroscopy from 2016 to 2020. We hypothesize that a small but increasing percentage of 
Table 1. The Demographics of Orthopaedic Surgery ACGME Case Log Respondents

\begin{tabular}{ccc}
\hline Year & $\begin{array}{c}\text { Total Number of } \\
\text { Residency Programs }\end{array}$ & $\begin{array}{c}\text { Total Number } \\
\text { of Residents }\end{array}$ \\
\hline 2016 & 153 & 705 \\
2017 & 156 & 709 \\
2018 & 154 & 729 \\
2019 & 154 & 725 \\
2020 & 154 & 724 \\
\hline
\end{tabular}

total knee arthroscopy case volume involves the pediatric population and that widespread variability in case exposure is present.

\section{Methods}

The ACGME case log reports for graduating orthopaedic surgery residents were reviewed from 2016 to 2020. The ACGME presents national averages of several procedures within particular anatomic categories. Procedures include incision, excision, intro or removal, repair/revision/ reconstruction, trauma, fracture/dislocation, manipulation, arthrodesis, amputation, arthroscopy, and other. Anatomic categories include shoulder, humerus/elbow, forearm/wrist, hand/fingers, pelvis/hip, femur/knee, leg/ ankle, and foot/toes. In this study, we assessed the average number of total (adult and pediatric), adult, and pediatric knee arthroscopy cases performed per resident listed under the "femur/knee" category year over year from 2016 to 2020 in order to calculate a percent change in case volume. In addition, we compared the ratio of the average number of procedures performed by the 10th and 90th percentiles of residents to determine case volume variability.

\section{Statistical Analysis}

The mean case volumes reported per resident were compared using unpaired two-tailed $t$ tests. Pearson $\chi^{2}$ analysis was used to compare fold differences among the 10th and 90th percentiles of graduating residents. The level of statistical significance was designated as $P<.05$. Excel software, version 16.0 (Microsoft Corp., Redmond, WA), was used for data input and statistical tests.

\section{Results}

The total number of orthopaedic surgery residency programs was 153 (705 residents) in 2016, 156 (709 residents) in 2017, 154 (729 residents) in 2018, 154 (725 residents) in 2019, and 154 (724 residents) in 2020 (Table 1).

The average number of total knee arthroscopy procedures performed per resident was $113.9 \pm 47$ in 2016, which decreased to $111 \pm 51$ in 2020, representing a $2.6 \%$ decrease $(P=.264)$ (Table 2$)$. The average number of adult knee arthroscopy procedures performed per resident was $100 \pm 45$ in 2016, which decreased to $94.6 \pm 47$ in 2020, representing a $5.4 \%$ decrease $(P<.027)$. The average number of pediatric knee arthroscopy procedures performed per resident was $13.9 \pm 10$ in 2016 , which increased to $16.4 \pm 13$ in 2020 , representing an $18 \%$ increase $(P<.005)$. Case volume trends for each of these categories from 2016 to 2020 are depicted in Fig 1.

The average number of total knee arthroscopy cases performed by the 10th percentile and 90th percentile of residents was 64 and 170 in 2016, representing a 2.66-fold difference, compared to 59 and 176 in 2020, representing a 2.98 -fold difference (Table 3 ). This increase in variability was not statistically significant $(P=.581)$. The average number of adult knee arthroscopy cases performed by the 10th and 90th percentile of residents was 55 and 151 in 2016, representing a 2.75 -fold difference, compared to 47 and 154 in 2020, representing a 3.28-fold difference. The increase in variability was not statistically significant $(P=.440)$. The average number of pediatric knee arthroscopy cases performed by the 10th percent and 90th percentile of residents was 3 and 26 in 2016, representing an 8.67-fold difference, compared with 3 and 33 in 2020, representing an 11 -fold difference. The increase in variability was not statistically significant $(P=.781)$. Trends in fold difference for each of these categories from 2016 to 2020 are depicted in Fig 2.

\section{Discussion}

Our study revealed that resident exposure to pediatric knee arthroscopy has significantly increased over the past 5 years. However, widespread variability in case volume is present and likely masked by the abundance of adult cases performed each year.

The ACGME mandates residents to perform a minimum of 30 knee arthroscopy cases each year. This expectation has been exceeded nearly 4 -fold in recent years, as residents averaged a total of 114 cases of knee arthroscopy per year from 2016 to 2020. No significant

Table 2. Mean Number of Knee Arthroscopy Procedures for Orthopaedic Surgery Residents in 2016 and 2020

\begin{tabular}{lccc}
\hline Demographic & 2016 & 2020 & $\%$ Change \\
\hline Total & $113.9 \pm 47[110$ to 117$]$ & $111 \pm 51[107$ to 115$]$ & $-2.6 \%$ \\
Adult & $100 \pm 45[96.7$ to 103$]$ & $94.6 \pm 47[91.2$ to 98$]$ & $-5.4 \%$ \\
Pediatric & $13.9 \pm 10[13.2$ to 14.4$]$ & $16.4 \pm 13[15.5,17.3]$ & $P=.264$ \\
\hline & & & $P<.027$ \\
\hline
\end{tabular}

Data are presented as means \pm SD [95\% confidence interval]. 


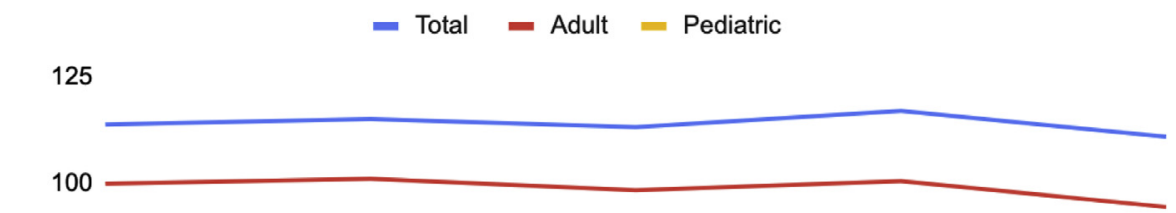

Fig 1. Trends in knee arthroscopy case volumes for graduating orthopaedic surgery residents from 2016 to 2020 .

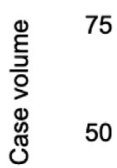

25 0 2016
2017 changes in case volume ( 114 in 2016 vs 111 in 2020; $P=$ .264) or fold difference between the 10th and 90th percentiles of residents (2.66 in 2016 and 2.98 in 2020; $P=.58$ ) occurred during this same time period. Although these data suggest that residents have achieved an adequate case volume for knee arthroscopy in recent years, important differences arise once adult and pediatric cases are individually assessed. The mean number of pediatric knee arthroscopy cases was 16.4 in 2020, which accounted for only $15 \%$ of a resident's overall case exposure to knee arthroscopy. In comparison, the mean number of adult and total (adult and pediatric) cases was 94.6 and 111 , respectively. Furthermore, wide variability in resident exposure to pediatric knee arthroscopy was present. As of 2020, an 11 -fold difference in case volume between the 10th and 90th percentiles of residents existed. Case volume variability was much lower for adult cases during this time, with a fold difference of 3.28, and even lower when total (adult and pediatric) cases were assessed, with a fold difference of 2.98. Thus, low case volumes and wide variability in resident exposure to pediatric knee arthroscopy seems to be masked by the abundance of adult cases.

While the discrepancy in resident exposure to knee arthroscopy is apparent, it is unclear how this correlates with procedural proficiency. Kohring et al. studied the perceptions of recently graduated orthopaedic residents on the ability to perform common orthopaedic procedures at the end of training and on the number of cases needed to achieve independence. ${ }^{2}$ With respect to knee arthroscopy, $96 \%$ of residents were comfortable performing meniscectomies independently, compared to $56.5 \%$ of residents for ACL reconstruction. ${ }^{2} 28.8 \pm$ 19.1 meniscectomies were recommended to achieve procedural independence, compared to $36.6 \pm 21.9$ ACL reconstructions. ${ }^{2}$ However, each of these findings pertained to adult cases specifically. The only pediatric knee arthroscopy procedure studied was irrigation and debridement. Ninety eight percent of residents were comfortable with independent practice of this procedure and recommended $16.6 \pm 14.2$ cases to achieve competency. $^{2}$ These findings suggest that resident confidence in performing independent knee arthroscopy is dependent on procedure type and patient demographic.

We showed that resident case volume for pediatric knee arthroscopy increased $18 \%$ over the past 5 years, from 13.9 in 2016 to 16.4 in $2020(P<.005)$, which parallels national trends in the rising incidence of pediatric knee arthroscopy. ${ }^{3}$ Expansion of this field is developing fast, thanks to advancements in arthroscopic technology, increasing surgeon comfortability, and growing

Table 3. Fold Difference in the Average Number of Knee Arthroscopy Procedures Performed by the 10th and 90th Percentile of Orthopaedic Surgery Residents

\begin{tabular}{|c|c|c|c|c|c|}
\hline Demographic & Year & 10th & 90th & Fold Difference & $P$ Value \\
\hline \multirow[t]{2}{*}{ Total } & 2016 & 64 & 170 & 2.66 & $P=.581$ \\
\hline & 2020 & 59 & 176 & 2.98 & \\
\hline Adults & 2016 & 55 & 151 & 2.75 & $P=.440$ \\
\hline \multirow[t]{2}{*}{ Pediatrics } & 2016 & 3 & 26 & 8.67 & $P=.781$ \\
\hline & 2020 & 3 & 33 & 11 & \\
\hline
\end{tabular}




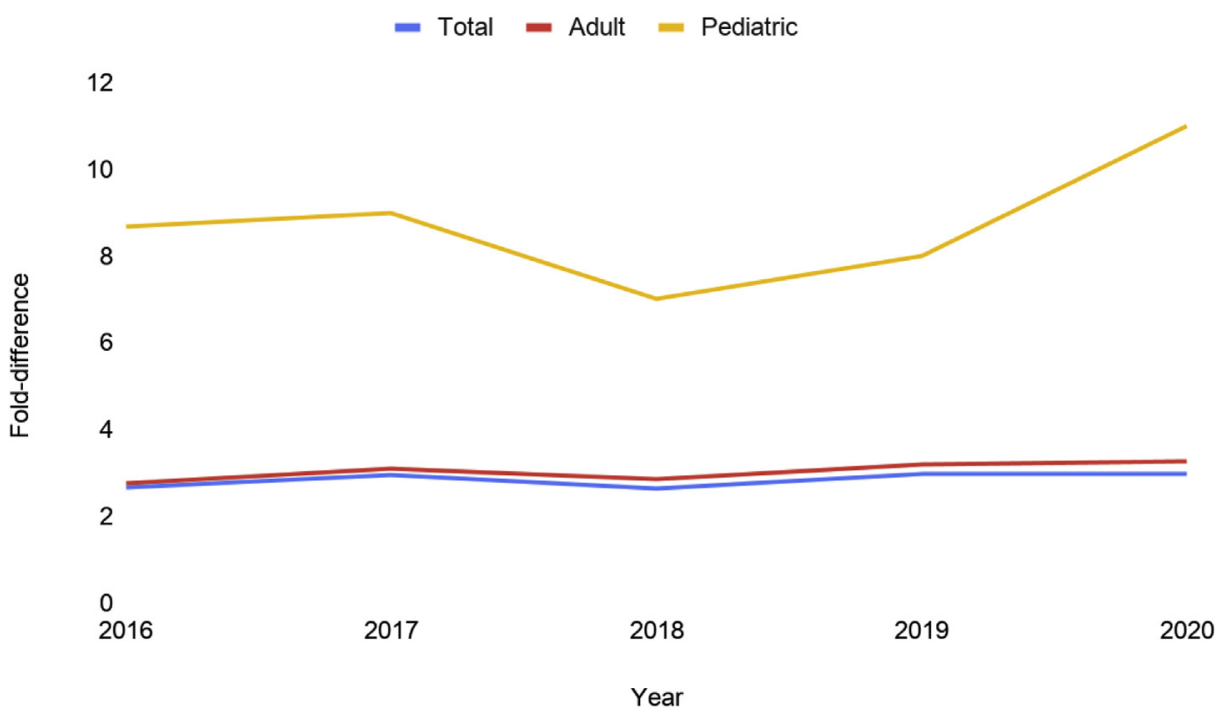

Fig 2. Trends in fold difference amongst the 10th and 90th percentile of resident-performed cases of knee arthroscopy from 2016 to 2020.

knowledge of child joint pathology. ${ }^{4}$ While knee arthroscopy is used in children and adolescents to treat intra-articular fractures, ${ }^{5}$ osteochondritis dissecans, ${ }^{6}$ discoid lateral meniscus, ${ }^{7}$ and synovitis, ${ }^{8}$ the most common indications include sport injuries of the meniscus and ACL. ${ }^{9}$

The incidence of acute and overuse sport injuries in pediatric athletes is at an all-time high, given the rise in sport participation. ${ }^{10}$ Approximately 45 million children and adolescents in the United States participate in organized sports, ${ }^{10}$ contributing to 2.6 million emergency room visits each year for sports-related injuries. ${ }^{11}$ In addition, increased sport specialization-defined as intensive, yearround participation in single-sport training-continues to grow in popularity, ${ }^{12}$ as emphasis on competitive success, elite-level travel team selection, collegiate scholarships, Olympic and National team membership, and professional contracts become widespread. ${ }^{13}$

The knee is the most reported site of musculoskeletal injury in pediatric athletes. ${ }^{9}$ Thus, it is no surprise that the largest application for arthroscopy in this population is the treatment of knee pathology. ${ }^{3}$ ACL reconstruction and meniscal repair account for a large majority of pediatric knee arthroscopy cases. ${ }^{9}$ While nonoperative or delayed surgical treatment of these injuries in the skeletally immature was once preferred to avoid physeal injury and risk of growth deformity, ${ }^{14}$ early arthroscopic management has taken favor. ${ }^{15} \mathrm{~A}$ recent systematic review showed excellent clinical outcomes in patients undergoing arthroscopic meniscal repair, regardless of the time from injury, location/ pattern of tear, or technique used. ${ }^{16}$ Similarly, ACL reconstruction within 12 weeks of injury is associated with a lower incidence of secondary meniscal injury and a higher rate of return to sports when compared to nonoperative management or delayed surgery. ${ }^{17}$
Technical differences among adult and pediatric knee arthroscopy exist and serve as important aspects of arthroscopy education for orthopaedic residents. The pediatric knee joint is smaller in size and involves neighboring growth plates, requiring adapted techniques with miniaturized equipment. ${ }^{4}$ Gentle exposure maneuvers (valgus and varus traction) must be used to avoid iatrogenic fracture ${ }^{4}$ and intraoperative awareness of the physes must be maintained to minimize risk of physeal arrest and limb length discrepancy. ${ }^{18}$ Unlike adult cases, the amount of residual limb growth should be documented preoperatively for pediatric patients. ${ }^{4}$ The proximal tibia and distal femur contribute to $6 \mathrm{~mm}$ and $10 \mathrm{~mm}$ of residual growth until the age of 13.5 in females and 15.5 in males. ${ }^{19}$ Given these anatomic considerations, the optimal approach of certain arthroscopic knee procedures remains controversial in skeletally immature patients. In the case of ACL reconstruction, reluctance to place drill holes across open physes has led to the development of "physeal-sparing" and "partial transphyseal" reconstruction techniques. ${ }^{20}$ Even in cases that avoid direct compromise of the physes, the graft may exert a "tenodesis effect" of tension on the growth plates. ${ }^{18}$ Similarly, in the case of fracture fixation, hardware should spare the physes when possible and be removed expeditiously. ${ }^{4}$

This study revealed significant variability in pediatric knee arthroscopy cases performed by orthopaedic surgery trainees. The findings presented in this study may assist in optimizing arthroscopy resident education. Perhaps existing ACL reconstruction and knee arthroscopy case minimum requirements should be updated to include a set number of pediatric cases. These changes might help reduce case volume variability and discrepancies in resident education. 


\section{Limitations}

There are several limitations of this study. First, the ACGME case log data do not specify the types of procedures (or indications of said procedures) within the knee arthroscopy category. Therefore, it was assumed that trends in case volume and variability for this generic category could be used to assume trends for specific procedure types and indications, such as arthroscopic meniscal repair and operative sport injury, respectively. Second, the accuracy of ACGME case log data has been questioned as a result of underreporting or overreporting among residents. ${ }^{21}$ Third, the degree of resident participation within each case is subject to reporting bias, which may also threaten the accuracy of the ACGME case log data.

\section{Conclusion}

Pediatric knee arthroscopy comprises a small yet growing percentage of knee arthroscopy case volume of graduating orthopaedic surgery residents. However, wide variability in resident exposure is present and likely masked by the abundance of adult cases performed each year.

\section{References}

1. Marsh JL, Potts JR 3rd, Levine WN. Challenges in resident education: Is the Next Accreditation System (NAS) the answer? AOA critical issues. J Bone Joint Surg Am 20 14;96:e75.

2. Kohring JM, Harrast JJ, Stotts AK, et al. Resident independence performing common orthopaedic procedures at the end of training: Perspective of the graduated resident. J Bone Joint Surg Am 2020;102:e2.

3. Siparsky PN, Kocher MS. Current concepts in pediatric and adolescent arthroscopy. Arthroscopy 2009;25:1453-1469.

4. Accadbled F. Arthroscopic surgery in children. Orthop Traumatol Surg Res 2010;96:447-455.

5. Accadbled F, N'Dele D. Arthroscopic treatment of pediatric fractures. J Pediatr Orthop 2018;38:S29-S32 (Suppl 1).

6. Bauer KL. Osteochondral injuries of the knee in pediatric patients. J Knee Surg 2018;31:382-391.

7. Kim JH, Ahn JH, Kim JH, Wang JH. Discoid lateral meniscus: importance, diagnosis, and treatment. J Exp Orthop 2020;7:81.

8. Sarpel Y, Ozkan C, Toğrul E, Tan I, Gülşen M. Arthroscopy of the knee in pre-adolescent children. Arch Orthop Trauma Surg 2007;127:229-234.
9. McConkey MO, Bonasia DE, Amendola A. Pediatric anterior cruciate ligament reconstruction. Curr Rev Musculoskelet Med 201 1;4:37-44.

10. Merkel DL. Youth sport: positive and negative impact on young athletes. J Sports Med 2013;4:151-160.

11. Burt CW, Overpeck MD. Emergency visits for sportsrelated injuries. Ann Emerg Med 2001;37:301-308.

12. Fabricant PD, Lakomkin N, Sugimoto D, Tepolt FA, Stracciolini A, Kocher MS. Youth sports specialization and musculoskeletal injury: A systematic review of the literature. Phys Sportsmed 2016;44:257-262.

13. DiFiori JP, Benjamin HJ, Brenner JS, et al. Overuse injuries and burnout in youth sports: A position statement from the American Medical Society for Sports Medicine. Br J Sports Med 2014;48:287-288.

14. Pressman AE, Letts RM, Jarvis JG. Anterior cruciate ligament tears in children: an analysis of operative versus nonoperative treatment. J Pediatr Orthop 1997;17: 505-511.

15. Werner BC, Yang S, Looney AM, Gwathmey FW Jr. Trends in pediatric and adolescent anterior cruciate ligament injury and reconstruction. J Pediatr Orthop 2016;36: 447-452.

16. Ferrari MB, Murphy CP, Gomes JLE. Meniscus repair in children and adolescents: A systematic review of treatment approaches, meniscal healing, and outcomes. J Knee Surg 2019;32:490-498.

17. James EW, Dawkins BJ, Schachne JM, et al. Early operative versus delayed operative versus nonoperative treatment of pediatric and adolescent anterior cruciate ligament injuries: A systematic review and meta-analysis. Am J Sports Med 2021. Mar 15:363546521990817.

18. Edwards TB, Greene CC, Baratta RV, Zieske A, Willis RB. The effect of placing a tensioned graft across open growth plates. A gross and histologic analysis. J Bone Joint Surg Am 2001;83:725-734.

19. Menelaus MB. Correction of leg length discrepancy by epiphysial arrest. J Bone Joint Surg Br 1966;48: 336-339.

20. Fabricant PD, Jones KJ, Delos D, et al. Reconstruction of the anterior cruciate ligament in the skeletally immature athlete: a review of current concepts: AAOS exhibit selection. J Bone Joint Surg Am 2013;95:e28.

21. Salazar D, Schiff A, Mitchell E, Hopkinson W. Variability in Accreditation Council for Graduate Medical Education Resident Case Log System practices among orthopaedic surgery residents. J Bone Joint Surg Am 2014;96:e22. 